\title{
Infestazione intestinale da Schistosoma mansoni: un caso emblematico di importazione
}

\author{
Maria Chiara Medori', Paolo Andreani', Cinzia Di Giuli², Gianni Pelini', \\ Alessandro Lavagna ${ }^{2}$ \\ Ospedale "S. Maria", Terni \\ 'Laboratorio di Analisi Chimico-cliniche e Microbiologia \\ ${ }^{2}$ Clinica di Malattie Infettive
}

\section{RIASSUNTO}

Viene descritto, sia dal punto di vista clinico che parassitologico, un caso di importazione di schistosomiasi intestinale da S. mansoni contratta durante un soggiorno in Tanzania. Dopo circa 50 giorni da un contatto casuale ma ripetuto con le acque del Lago Vittoria, al soggetto, giovane adulto in missione umanitaria, compare febbre elevata, accompagnata da astenia (presente da più giorni) cui segue un episodio di diarrea acuta. La comparsa anche di una ipereosinofilia, dapprima assente, spinge il curante ad eseguire immediatamente un esame coproparassitologico standard (in precedenza sempre negativo) che risulta positivo per uova di S. mansoni. La terapia condotta con praziquantel risolve il caso e porta a guarigione il soggetto, cui inizialmente era stata esclusa la malaria, allorché dopo circa I mese dal rientro aveva presentato rialzo termico con disturbi respiratori.

\section{INTRODUZIONE}

La schistosomiasi è causata da trematodi del genere Schistosoma, elminti a complesso ciclo vitale, che da adulti vivono nei vasi sanguigni dell'uomo, e che sono gli unici, tra i "digenei" di interesse medico, a presentare sessi separati (5). Le tre specie principali che infestano l'uomo sono Schistosoma haematobium, Schistosoma mansoni e Schistosoma japonicum (6). S. haematobium causa la schistosomiasi (bilharziosi) urinaria; $S$. mansoni e $S$. japonicum (al pari di $S$. mekongi e $S$. intercalatum) causano la schistosomiasi intestinale $(2,5,6)$.

Schistosoma mansoni è presente soltanto nel continente africano (africa subsahariana e regione del Nilo) e nel sud-america (Brasile e regioni limitrofe), e si stimano essere in svariate decine di milioni le persone colpite da questa parassitosi, con almeno 10-20 milioni di soggetti con infestazione grave con coinvolgimento epato-splenico $(3,6)$.

Il ciclo vitale prevede l'eliminazione delle uova con le feci in ambienti di acqua dolce, da cui si libera il miracidio che viene ingerito da una lumaca del genere Biomphalaria, nella quale si ha lo sviluppo sino allo stadio di cercaria (3).

La cercaria viene eliminata nell'acqua, da cui penetra per via transcutanea nell'ospite definitivo che si immerga in tali acque, per migrare sino ai vasi linfatici mesenterici ove matura in verme adulto, differenziandosi in maschio e femmina, ove può vivere per molti anni $(3,5,6)$. Dall'accoppiamento vengono prodotte uova che vengono espulse nel canale intestinale ed eliminate con le feci, essendo il periodo di prepatenza di circa 4-8 settimane $(4,5,6)$.

L'infestazione può occorrere anche dopo un bre- vissimo bagno in acque infestate, così come dopo un banale attraversamento a piedi di un corso d'acqua in cui siano presenti i molluschi infestati (l'ospite intermedio), talché spesso, nel soggetto di ritorno da zone endemiche, può sfuggire l'elemento anamnestico chiave che può indirizzare verso un giusto percorso diagnostico $(4,6)$.

In tale presentazione riportiamo così un caso di schistosomiasi di importazione in un giovane adulto, caso del tutto emblematico, sia nella sua particolarità di presentazione ed evoluzione, sia nella sua multidisciplinarietà diagnostica $(1,6)$.

\section{DESCRIZIONE DEL CASO}

All'inizio dell'estate 2004, un adulto di anni 28, di nazionalità italiana, di professione accompagnatore turistico e di vocazione operatore sanitario, si reca, con altri collaboratori, in Tanzania, nella regione Mara (in prossimità del lago Vittoria), per la costruzione di un dispensario in un villaggio lì ubicato, all'interno di un progetto di cooperazione. Tale soggetto si era già recato altre volte in tale regione ed aveva sempre praticato profilassi antimalarica, ma non in questa circostanza.

Il soggetto riferirà di essersi bagnato più volte nel lago al fine di raccogliere acqua da portare al villaggio da utilizzare per lavarsi (tale acqua verrà sempre lasciata al sole 48 ore prima di essere poi utilizzata a tale scopo), anche se riferirà di avere sempre indossato pantaloni e stivali per proteggersi, sapendo, probabilmente, dei rischi intrinseci.

Dopo 10 giorni dall'arrivo, al soggetto compare diarrea (4-5 scariche nell'arco di alcune ore) e febbre elevata, ma dopo meno di 12 ore tale sin- 
tomatologia scompare spontaneamente. Il soggetto soggiorna una ventina di giorni in Africa e poi rientra in Europa per recarsi peraltro direttamente in Austria.

Dopo circa 1 mese dal primo episodio, al soggetto ricompare febbre con brivido, accompagnata da cefalea e sintomatologia respiratoria.

Gli viene somministrata così una terapia per os con claritromicina ( $250 \mathrm{mg} \times 2 /$ die). Dopo altri 2 giorni rientra in Italia e, persistendo la febbre, si ricovera in Clinica di Malattie Infettive per sospetta malaria.

Al momento del ricovero vengono fatti esami ematochimici di routine, ricerca plasmodi malarici su strisci periferici di sangue, ECG, Eco-addome, e radiografia del torace.

Quest'ultima evidenzia un quadro compatibile con esiti di processo flogistico in via di risoluzione, mentre le altre indagini strumentali risultano negative, così come la ricerca di plasmodi malarici (striscio sottile e goccia spessa), mentre le indagini ematochimiche evidenziano soltanto la presenza di una piastrinopenia (90.000 piastrine/microlitro).

Tali ed ulteriori esami ematochimici e strisci sottili con gocce spesse (per escludere con sicurezza una malaria) venivano ripetuti il giorno successivo, sempre con esito negativo.

Viene infatti confermata la sola piastrinopenia. Anche una serie di emocolture risulteranno negative.

Viene evidenziata esclusivamente la presenza di agglutinine a freddo e una positività per anticorpi IgG anti-Mycoplasma pneumoniae (ma con IgM negative).

Viene sospesa la claritromicina ed iniziata una terapia con doxiciclina (100 mg x 2/die). Dopo 24 ore (ovvero al terzo giorno di ricovero), il soggetto, apiretico e in buone condizioni generali (a parte una residua tosse secca) decide di firmare le dimissioni volontarie per partire per un viaggio di 10 giorni in Austria e Repubblica Ceca.

Al suo rientro in Italia (circa 50 giorni dopo l'ini-

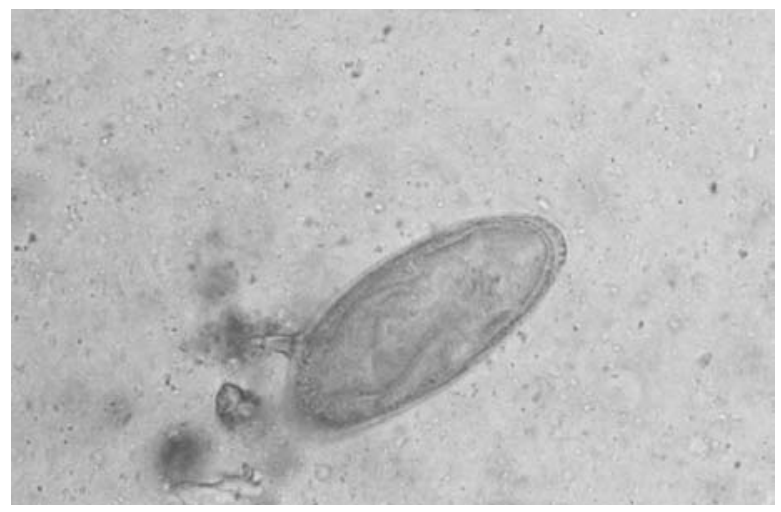

Figura I. Uovo di S. mansoni (M. O. dopo FEA, $40 \times$ 10) zio del viaggio in Tanzania), al soggetto ricompare febbre $\left(38.5-39^{\circ} \mathrm{C}\right)$, accompagnata da marcata astenia, e diarrea (2-3 scariche/die con feci liquide, senza muco né sangue).

Pratica a domicilio una terapia con tachipirina e dissenten.

Persistendo la sintomatologia, dopo altri 2 giorni si reca al DH della Clinica di Malattie Infettive; viene visitato e viene sottoposto ad alcuni accertamenti ematochimici: emocromo, Widal-Wright, esame coproparassitologico.

Risulta tutto negativo; le piastrine sono nei limiti inferiori della norma (145.000/microlitro). Gli viene prescritta una terapia con ciprofloxacina (500 mg x 2/die).

Dopo 2 giorni si arresta la diarrea, ma persistono febbre elevata, astenia e ricompare una tosse secca. Viene così ricoverato in reparto, e, sospesa la terapia antibiotica, viene sottoposto a Rx-torace (sarà negativo), a visita ORL (negativa), indagini ematochimiche.

Le piastrine sono $155.000 /$ microlitro, ma la conta degli eosinofili sale al 31.9\%, accompagnata da un aumento dei seguenti indici epatici: AST 94 UI/litro, ALT 213 UI/litro, gammaGT 200 UI/litro e VES pari a 78.

Viene immediatamente richiesto un altro esame coproparassitologico standard (verranno eseguite 3 raccolte di feci in 3 giorni successivi) e nel frattempo viene prescritta una terapia a base di metronidazolo (500 $\mathrm{mg} \mathrm{x} \mathrm{3/die).}$

Mentre la colorazione di Giemsa, condotta sui tre campioni fecali, da esito negativo, già l'osservazione diretta microscopica in soluzione fisiologica mette in evidenza la presenza di uova non opercolate, con spina laterale, di dimensioni di 60 x $150 \mu \mathrm{m}$, chiaramente identificabili come uova di Schistosoma mansoni (figura I).

L'arricchimento formolo-etilacetato (SEDCONNECT ${ }^{\mathrm{TM}}$, Medical Chemical Corporation) conferma la presenza di numerose uova di $S$. mansoni (figura II) nonché la presenza di cristalli di Charcot-Leyden, ben correlabile alla ipereo-

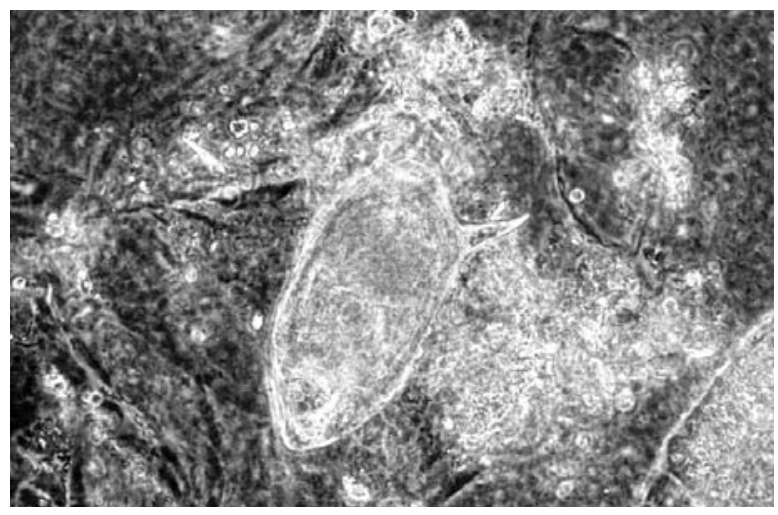

Figura II. Uovo di S. mansoni (M. O. in contrasto di fase, $40 \times 10$ ) 
sinofilia.

Viene pertanto fatta diagnosi di schistosomiasi intestinale e viene prescritta una terapia a base di praziquantel. In attesa dell'arrivo del farmaco specifico (la terapia sarà inziata 5 giorni dopo) vengono ripetuti gli esami ematochimici (3 giorni dopo) che danno i seguenti esiti: ipereosinofilia (45.0\%), AST 82 UI/litro, ALT 197 UI/litro, gammaGT 556 UI/litro, LDH 492 UI/litro.

Il soggetto verrà dimesso, apiretico, con ecografia epatica nella norma, con eosinofilia scesa al $30.0 \%$.

A distanza di 3 mesi circa viene eseguito un esame coproparassitologico di controllo (su 3 campioni), che darà esito negativo, sia dopo arricchimento formolo-etilacetato sia dopo esecuzione della tecnica di Kato-Katz.

La conta degli eosinofili è del 7.5\%, gli indici epatici risultano AST $26 \mathrm{UI} /$ litro, ALT $24 \mathrm{UI} /$ litro, gammaGT 27 UI/litro, LDH 257 UI/litro.

\section{COMMENTO}

Nonostante le apparenti attenzioni prese, sembra sia stato inevitabile un contatto, in questa circostanza verosimilmente attraverso gli arti superiori, con le acque di un bacino idrico (in tal caso il Lago Vittoria, Africa Centrale) notoriamente a rischio per schistosomiasi $(3,6)$.

Correttamente esclusa una malaria, giustamente temuta anche per l'assenza di una profilassi adeguata, dopo pochi giorni dal rientro dalla Tanzania in Europa, su motivato quadro clinico (forse imputabile ad una polmonite da batteri atipici), viene sospettata, a distanza di quasi 2 mesi dal soggiorno nel paese africano in questione, una parassitosi intestinale, e, nello specifico, una febbre di Katayama, non di rado riscontrabile come prima espressione della schistosomiasi in soggetti non residenti in aree endemiche (6), sulla base del quadro clinico presentatosi, sì da richiedere immediatamente un esame coproparassitologico standard (1).

Sicuramente la stretta collaborazione tra infettivologo e microbiologo ha permesso un pronto ed efficace intervento diagnostico, grazie anche alla conoscenza anamnestica di un viaggio in paese endemico per tali parassitosi.

L'emblematicità del caso importato è legato agli aspetti epidemiologici correlati, alla evoluzione clinica dell'infestazione (la febbre di Katayama è la forma acuta di esordio della schistosomiasi ed è associata alla iniziale produzione massiccia di uova con febbre alta, ipereosinofilia, coinvolgimento epatico, diarrea), al corretto approccio diagnostico anche consequenziale e motivabile dalla attenta anamnesi recente del soggetto coinvolto $(1,3,5)$.

\section{Ringraziamenti}

Si ringrazia il Dr. Daniele Crotti dell'Ospedale "R. Silvestrini" di Perugia per i suggerimenti forniti e per la revisione critica del manoscritto.

\section{BIBLIOGRAFIA}

1. Bernieri F, Crotti D, Galli D, Raglio A. Manuale illustrato di diagnostica parassitologica. Edizioni Selecta Medica, Pavia, 2001.

2. Cancrini G. Parassitologia medica illustrata. Lombardo Editore, Roma, 1996, 165-176.

3. Chiodini PL, Moody AH, Manser DW. Atlas of Medical Helminthology and Protozoology, Churchill Livingstone, Harcourt Publishers Limited, London, IV Edition, 2001, 27-29.

4. Crotti D, Del Sante M, Fonzo G, Gebremariam T. Schistosomiasi e parassitosi intestinali nella popolazione scolare dell'area di Mendefera, Eritrea. G It Med Trop, 1999; Vol. 4, N. 1-2: 15-19.

5. De Carneri I. Parassitologia generale e umana. Casa Editrice Ambrosiana, Milano, XI Edizione, 1992, 185-192.

6. Garcia LS. Diagnostic Medical Parasitology. ASM Press, Washington DC, IV Edition, 2001, 445-476.

Maria Chiara Medori

Viale della Stazione, 66 05100 Terni

Tel.: 0744424315

E-mail: mariachiara.medori@libero.it 